\begin{tabular}{l|r} 
SISTEMA \\
ELETRÔNICO \\
DE REVISTAS \\
SER I UfPR
\end{tabular}

\title{
Influência de fatores socioeconômicos sobre os crimes ambientais no Distrito Federal
}

\section{Influence of socioeconomic factors on environmental crimes in the Brazilian Federal District}

\author{
Renata Esteves RIBEIRO ${ }^{1 *}$, Rodrigo Studart CORREA ${ }^{1}$ \\ ${ }^{1}$ Universidade de Brasília (UnB) - Campus Planaltina, DF, Brasil. \\ *E-mail de contato: renataeribeiro@hotmail.com
}

Artigo recebido em 2 de fevereiro de 2018, versão final aceita em 11 de março de 2019.

RESUMO: Danos e problemas ambientais são construídos por processos complexos, constituídos por uma combinação de fatores materiais e sociais. Diferentes escritores da criminologia verde, ramo que foca questões referentes a danos, transgressões e crimes ambientais, alocam os debates em torno das questões relacionadas à pobreza, falta de saúde, corrupção e transgressões corporativas. Seguindo esse entendimento, este trabalho objetivou i) verificar a influência de variáveis socioeconômicas sobre os crimes ambientais registrados pela da Polícia Civil do Distrito Federal entre os anos de 2013 a 2016 e ii) determinar se as teorias da criminologia tradicional se aplicam aos crimes ambientais. Dados socioeconômicos disponíveis na Pesquisa Distrital por Amostra de Domicílios e os registros de crimes ambientais ocorridos no Distrito Federal (DF) foram submetidos à análise de regressão multivariada e à análise de variância para determinar o modelo mais eficiente para explicar a relação entre esses dois conjuntos dados. Os resultados indicaram que a faixa etária entre 40 e 59 anos, os residentes que imigraram para o DF após o ano 2000 e a renda per capita foram as variáveis que mais contribuíram para explicar o número de registros de crimes ambientais no DF. Os resultados sugerem que contextos de privação social e econômica não são os únicos determinantes para a ocorrência de crimes ambientais no DF. A frequente relação com as variáveis relacionadas à imigração aponta para a aplicação da teoria da desorganização social como uma das principais causas dos crimes registrados, pela incapacidade de a comunidade se organizar em prol do controle comunitário.

Palavras-chave: crimes ambientais; Polícia Civil do Distrito Federal; criminologia verde; regressão linear multivariada.

ABSTRACT: Environmental damages and problems arise from complex processes made up of material and social factors. 
Different authors of green criminology, a branch that focuses on issues related to damages, transgressions and crimes against nature, advocate a conceptual approach related to poverty, poor health, corruption and corporate transgressions. Following such understanding, this study aimed to: i) check the influence of socioeconomic variables on environmental crimes recorded by the Brazilian Federal District Police between 2013 and 2016, and (ii) determine whether theories of traditional criminology apply to environmental crimes in the Brazilian Federal District - BFD. Socioeconomic data available in the District Household Sample Survey and records on environmental crime in BFD were subjected to multivariate regression and analysis of variance. Results indicated that people age range between " 40 and 59 years old", residents who arrived in BFD after 2000 and per capita income were the variables that better explained environmental crime records. Results suggest that social and economic deprivation were not decisive to explain the occurrence of environmental crimes in BFD. A frequent relation between immigration and crime records points towards the theory of social disorganization, caused by community inability to organize themselves for the establishment of community self-controls.

Keywords: environmental crimes; Brazilian Federal District Police; green criminology; multivariate linear regression.

\section{Introdução}

As questões ambientais não existem de forma isolada da sociedade humana. Danos e problemas ambientais são resultados de processos complexos. Para compreender como os problemas ambientais são construídos, é necessário afirmar que esse processo incorpora elementos objetivos e subjetivos, constituídos por uma combinação de fatores materiais e sociais (White, 2008).

A palavra "criminologia", etimologicamente, vem do latim crimino (crime) e do grego logos (estudo, tratado) e denomina a ciência empírica e interdisciplinar que tem por objeto de análise o crime, o autor do comportamento delitivo, a vítima e o controle social das condutas criminosas, bem como a gênese, dinâmica e variáveis principais do crime, contemplando-o como problema individual e social (Molina \& Gomes, 2007).
No que se refere ao delito, a criminologia utiliza-se de um processo de verificação que analisa a conduta antissocial, suas causas geradoras e o tratamento dado ao delinquente, visando a prevenir a reincidência e entender falhas de prevenção (Penteado Filho, 2012).

Agrupando as teorias mais discutidas por sociólogos na definição das causas do crime e da violência, Beato Filho (1998) delimitou duas linhas: uma defende que criminalidade e violência são fenômenos originados essencialmente de fatores econômicos. A privação de oportunidades, a desigualdade social e a marginalização seriam estímulos decisivos para o comportamento criminoso (Parker \& Smith, 1979; Taylor et al., 1980). Outra linha defende que um baixo grau de integração moral produziria o fenômeno do crime, creditando ao delinquente e aos atos criminosos uma agressão ao consenso moral e normativo da sociedade

${ }^{1}$ Original de 1978 
(Durkheim, 2010)1. Procurando explicar as taxas de crimes violentos, sociólogos e criminólogos aplicaram diferentes técnicas estatísticas na análise de variáveis sociais, demográficas e econômicas para desenvolverem e defenderem suas teorias (Beato Filho, 1998).

O desenvolvimento da criminologia verde, ramo que foca especificamente em questões referentes a danos, transgressões e crimes contra o planeta, o ambiente natural e a diversidade de espécies não humanas e a própria espécie humana, tem levado a novos interesses, novos conceitos e novas técnicas de análise. Diferentes autores possuem ideias distintas sobre qual seria a maneira mais apropriada para se analisar o ambiente natural e o crime. Para alguns é importante considerar as particularidades dos problemas ambientais do ponto de vista criminológico, que incluem itens como transporte de lixo tóxico, fornecimento de água não potável, entre outros. Outros defendem uma abordagem mais conceitual, no sentido de alocar os debates em torno da interligação entre questões sociais e ambientais, como aquelas relacionadas à pobreza, saúde, corrupção, transgressões corporativas, reconhecendo a necessidade de uma abordagem multidisciplinar, envolvendo a cooperação entre diferentes especia- listas e diferentes áreas de conhecimento acadêmico (White, 2008).

No Brasil, a tutela dos bens ambientais conta com o auxílio de instrumentos oriundos do direito penal, do qual deve-se lançar mão somente como ultima ratio, quando as demais instâncias de responsabilização - civil e administrativa - já não se mostram mais suficientes para coibir a conduta danosa ao ambiente. Assim, a legislação ambiental brasileira volta-se preponderantemente à prevenção do dano ambiental e à sua reparação tempestiva e integral (Milaré, 2014).

Em face do exposto, este trabalho tem como objetivos i) verificar a influência das variáveis socioeconômicas sobre os registros de crimes ambientais feitos pela Polícia Civil do Distrito Federal e ii) determinar se teorias da criminologia se aplicam aos crimes ambientais estudados neste trabalho.

\section{Materiais e métodos}

Foi disponibilizada pela Polícia Civil do Distrito Federal (PCDF) uma planilha contendo os dados não sigilosos de 2.283 registros de crimes ambientais entre os anos de 2013 e 2016 que foram enquadrados na Lei de Crimes Ambientais (Lei

TABELA 1 - Registros de crimes ambientais por tipo penal definido na Lei n. ${ }^{\circ}$ 9.605/1998 e por ano de registro.

\begin{tabular}{llllll}
\hline & $\mathbf{2 0 1 3}$ & $\mathbf{2 0 1 4}$ & $\mathbf{2 0 1 5}$ & $\mathbf{2 0 1 6}$ & Total \\
\hline Dano & 252 & 146 & 226 & 237 & $861(37,7 \%)$ \\
\hline Fauna & 161 & 141 & 186 & 247 & $735(32,2 \%)$ \\
\hline Flora & 13 & 5 & 7 & 20 & $45(2 \%)$ \\
\hline Poluição & - & 1 & - & - & $1(<1 \%)$ \\
\hline Urbano & 149 & 132 & 162 & 198 & $641(28,1 \%)$ \\
\hline Total & $\mathbf{5 7 5}(\mathbf{2 5 , 2 \% )}$ & $\mathbf{4 2 5}(\mathbf{1 8 , 6 \% )}$ & $\mathbf{5 8 1}(\mathbf{2 5 , 5 \% )}$ & $\mathbf{7 0 2} \mathbf{( 3 0 , 8 \% )}$ & $\mathbf{2 . 2 8 3}$ \\
\hline
\end{tabular}


n. ${ }^{\circ}$ 9.605/1998) (Brasil, 1998). Os dados foram agrupados em cinco categorias de crimes ambientais (crimes totais, crimes contra a fauna, crimes contra a flora, dano a unidades de conservação e crimes contra o ordenamento urbano e o patrimônio cultural), de acordo com as seções existentes no capítulo "Dos crimes contra o Meio Ambiente" da referida Lei (Tabela 1). A seção "Dos Crimes contra a administração ambiental" não foi contemplada neste estudo, uma vez que os dados referentes a esse gênero de crime não foram disponibilizados pela PCDF.

Destaca-se que apesar de o crime de dano a unidades de conservação ser um tipo penal específico dentro da seção "Dos crimes contra a flora" (Brasil, 1998), pelo volume de ocorrências (861 registros daquele, contra 45 deste), optou-se por agrupá-los em uma categoria à parte, para uma melhor análise de sua incidência. Já o crime de poluição foi descartado da análise, por possuir apenas um registro na série temporal analisada.

Os dados socioeconômicos, usados como variáveis independentes neste trabalho, foram obtidos na Pesquisa Distrital por Amostra de Domicílios (PDAD) realizada pela Companhia de Planejamento do Distrito Federal - Codeplan (CODEPLAN, 2014; 2016). Ao todo, foram selecionadas 19 variáveis socioeconômicas dentre as disponíveis, correspondentes às variáveis utilizadas por Andrensen (2011) em seu trabalho sobre agregação local de crimes. Para fins de análise das variáveis mais tradicionalmente contempladas nas teorias criminais clássicas, os dados de "nível de escolaridade" foram somados, formando uma única variável, "nível pós-secundário", que agrega a parcela da população com níveis de escolaridade de "superior incompleto" a "doutorado". A seguir, destacam-se as razões para a seleção das variáveis utilizadas:

Distribuição populacional por sexo: análises realizadas por Hirschi \& Gottfredson (1983), baseadas na teoria da atividade de rotina, definiram a combinação das variáveis sexo e faixa etária como importantes preditoras de crimes. Uma vez que os dados da PDAD não oferecem essas variáveis de forma conjunta, optou-se por analisá-las separadamente.

Composição etária: considerada um fator preditivo segundo a teoria da atividade de rotina, a composição etária seguiu a estratificação disponível na PDAD. Hirschi \& Gottfredson (1983) avaliaram indivíduos a partir dos 15 anos de idade, mas destaca-se aqui que a idade penal mínima para imputação criminal no Brasil é de 18 anos. Alguns estudos avaliaram a influência da composição etária de forma indireta, utilizando variáveis compostas como o Índice de Desenvolvimento Humano (IDH), longevidade, educação e renda (Beato Filho, 1998; Beato \& Reis, 2000). As faixas etárias avaliadas neste trabalho foram: i) de 15 a 18 anos; ii) de 19 a 24 anos; iii) de 25 a 39 anos; iv) de 40 a 59 anos; v) de 60 a 64 anos; vi) 65 anos ou mais.

Estado civil/ união conjugal: ainda conforme a teoria da atividade de rotina, o estado conjugal dos habitantes constitui um fator preditivo à ocorrência de crimes pela sua influência nos alvos potenciais de vitimização e na capacidade de tutela da comunidade.

Imigração recente: Shaw \& McKay (2014), em seu trabalho clássico que deu origem à teoria da desorganização social, argumentam que três fatores estruturais - baixo status econômico, heterogeneidade étnica e mobilidade residencial-levam a um rompimento na organização da comunidade 
(Sampson \& Groove, 1989). Para expressar essa variável, optou-se por trabalhar com o percentual de imigrantes que se estabeleceram no Distrito Federal após o ano 2000, dados de imigração mais recentes fornecidos pela PDAD.

População com menos de um ano de moradia na região atual: a rotatividade populacional é uma das variáveis avaliadas por Andresen (2011), por impactar a capacidade da comunidade em se organizar em prol do controle comunitário. Kasarda \& Janowitz (1974) argumentam que a assimilação dos novatos pela comunidade local é uma questão temporal, operando como uma barreira ao desenvolvimento das redes de colaboração.

População com ensino pós-secundário: entre os estudos mais recentes desenvolvidos sobre a teoria da desorganização social, o nível de escolaridade é uma das formas de se avaliar a privação social e econômica de uma população (Beato Filho, 1998; Beato \& Reis, 2000; Batella \& Diniz, 2010). A relação entre nível educacional e criminalidade pode ser avaliada de forma direta, pelos dados de escolaridade e os crimes associados à comunidade em questão. Indiretamente, essa relação pode ser avaliada por meio de outras variáveis, como IDH e Coeficiente de GINI na educação (Beato Filho, 1998; Beato \& Reis, 2000; Batella \& Diniz, 2010). A educação formal torna-se uma pré-condição para o acesso do indivíduo a uma melhor qualificação profissional e, consequentemente, a uma maior renda (Batella \& Diniz, 2010).

Renda per capita média mensal: variável avaliada pela teoria da atividade de rotina, impactando a vulnerabilidade de alvos em potencial. Beato Filho (1998) afirma que, ao contrário do proposto por diversos trabalhos, a explicação mais correta para o crime não seria a pobreza, mas a riqueza, porque ela oferece alvos mais viáveis e compensadores. Ambientes mais prósperos seriam vistos como sinônimos de oportunidade para a ação criminosa.

Renda familiar média mensal: assim como renda per capita, a renda média familiar é uma variável considerada em estudos de teoria da desorganização social e de atividade de rotina.

Percentual de imóveis alugados: analisada conforme a teoria da desorganização social e da atividade de rotina, locatários tendem a ser mais transientes que os proprietários de imóveis.

Os dados de crimes ambientais e as variáveis socioeconômicas foram organizados e tratados utilizando o programa Microsoft Excel ${ }^{\circledR}$. Os dados socioeconômicos da PDAD são publicados a cada biênio, não sendo possível individualizar essas variáveis por ano. Optou-se, então, por trabalhar com a média dos biênios 2013/14 e 2015/16. Nesse mesmo sentido, os dados de crimes ambientais também tiveram suas médias calculadas para os dois biênios do estudo e, posteriormente, foram padronizados em razões por 100 mil habitantes.

Foi realizada uma correlação bivariada entre as variáveis independentes, utilizando o programa Statistica ${ }^{\circledR}$, com o objetivo de determinar o nível de dependência entre elas. As variáveis com alto coeficiente de correlação foram descartadas das análises posteriores, evitando assim a colinearidade nos resultados.

Utilizando o programa $R \AA$, os dados de variáveis dependentes e independentes foram normalizados conforme a equação a Eq. 1 (Legendre \& Legendre, 2012):

$$
\mathrm{Yi}^{\prime}=\log (\mathrm{Yi}+1)
$$


Os dados transformados foram submetidos à regressão linear multivariada para determinar como as variáveis socioeconômicas influenciam a ocorrência de crimes ambientais. Um procedimento stepwise foi realizado para determinar um submodelo de regressão, utilizando o valor k padrão igual a $2(\alpha=0,843)$. Esse procedimento é recomendado em estudos empíricos, em que se pretende reduzir o número de variáveis exploratórias para simplificar o conjunto de dados, selecionando apenas aquelas que contribuem mais significativamente (Legendre \& Legendre, 2012). Os resíduos das análises foram submetidos a teste de normalidade, pressuposto para a regressão, e os modelos de regressão pré e pós-stepwise foram submetidos à análise de variância

TABELA 2 - Correlação bivariada, variáveis socioeconômicas, Regiões Administrativas, Distrito Federal, Brasil. *p<0,05.

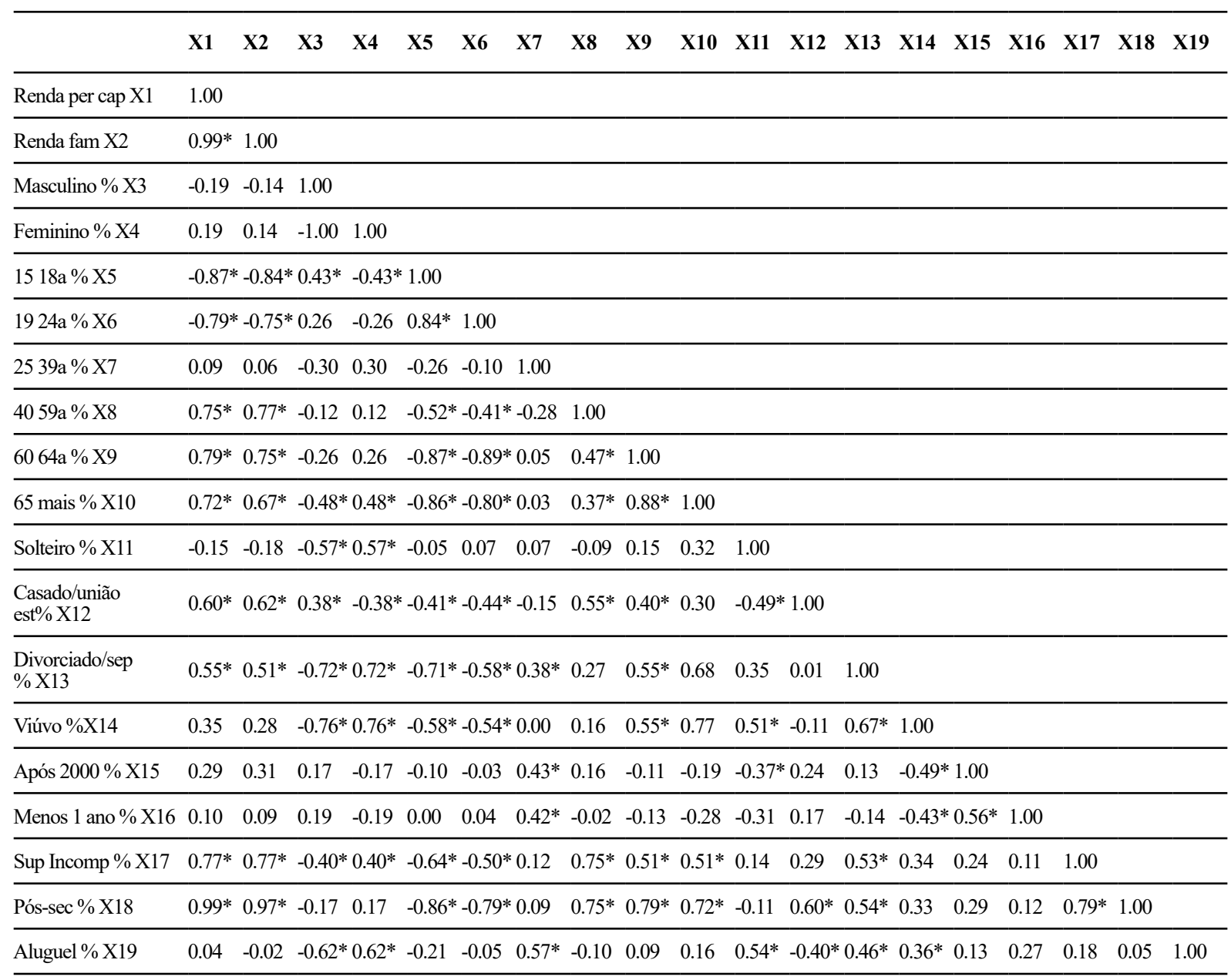


(Anova) para avaliação da significância da diferença entre eles (Gotelli \& Ellison, 2011).

\section{Resultados e discussão}

Após testar a correlação de Spearman, apenas alguns coeficientes foram superiores a 0,9 , com nível de significância $<0,05$ (Tabela 2). Baseado nesses resultados, optou-se por retirar das variáveis socioeconômicas os dados de "renda familiar" e "sexo feminino", fortemente correlacionados com "renda per capita" e "sexo masculino", respectivamente.
Após submeter os dados de crimes ambientais à regressão linear multivariada seguida de um procedimento stepwise, os resíduos de todas as regressões obtidas se mostraram normais e as análises de variância resultaram em diferenças não significativas entre os modelos regressores (Tabela 3). Desses resultados, depreende-se que o modelo mais eficiente é aquele que contempla menor número de variáveis, diminuindo, assim, a inserção de erros vinculados à obtenção delas.

Avaliando-se os resultados como um conjunto, com o objetivo de determinar a possibilidade de aplicação das teorias criminais clássicas aos crimes ambientais no Distrito Federal (DF), as variáveis

TABELA 3 - Análise de variância (Anova) entre as regressões lineares multivariadas.

\begin{tabular}{llllll} 
Anova & Dano & Fauna & Flora & Urbano & Total \\
\hline $\mathbf{F}$ & 0.0391 & 0.1059 & 0.1675 & 0.1152 & 0.0965 \\
\hline $\operatorname{Pr}(>\mathbf{F})$ & 1 & 0.9983 & 0.9918 & 0.996 & 0.9987 \\
\hline
\end{tabular}

TABELA 4 - Estatística $\mathrm{R}^{2}$ e $\mathrm{F}$ de cada regressão calculada para cada um dos cinco tipos de crimes ambientais. (1)Regressão pré-stepwise; (2) Regressão pós-stepwise. ${ }^{*} \mathrm{p}<0.1 ;{ }^{*} \mathrm{p}<0.05 ;{ }^{* * *} \mathrm{p}<0.01$.

\begin{tabular}{|c|c|c|c|c|c|c|c|c|c|c|}
\hline \multirow[b]{2}{*}{ Regressão } & \multicolumn{2}{|l|}{ Dano } & \multicolumn{2}{|l|}{ Fauna } & \multicolumn{2}{|l|}{ Flora } & \multicolumn{2}{|c|}{ Urbano } & \multicolumn{2}{|l|}{ Total } \\
\hline & 1 & 2 & 1 & 2 & 1 & 2 & 1 & 2 & 1 & 2 \\
\hline R2 Ajustado & -0.195 & 0.243 & 0.325 & 0.555 & 0.262 & 0.496 & 0.164 & 0.423 & -0.081 & 0.291 \\
\hline Estatística $\mathbf{F}$ & 0.713 & $2.068^{*}$ & 1.849 & $5.155^{*}$ & 1.626 & $4.279 *$ & * 1.347 & $3.201 * *$ & 0.868 & $2.369 * *$ \\
\hline
\end{tabular}


socioeconômicas que mais contribuíram para a ocorrência de crimes ambientais foram:

- Residentes na região após 2000: 3 vezes $(+)$;

- Renda per capita: 3 vezes (-);

- Faixa etária entre 40 e 59 anos: 3 vezes (-);

- Divorciados/separados: 2 vezes (-);
- Viúvos: 2 vezes (+);

- Residentes na região a menos de um ano: 2 vezes (-).

As variáveis socioeconômicas que influenciaram diretamente o aumento do número de registros

TABELA 5 - Regressão linear multivariada, total crimes ambientais/100 mil habitantes, Distrito Federal, Brasil.

\section{Total/100mil}

\section{Renda per capita}

19-24a \%

40-59a \%

\section{b (erro padrão)}

$$
-2.116^{* *}(0.779)
$$

$$
2.012(1.598)
$$

$-3.884 *(2.007)$

Casado/união est.

$$
3.527 *(2.005)
$$

\begin{tabular}{ll}
\hline Divorciado/separado & $-1.370(0.873)$ \\
\hline Viúvo & $2.338^{* *}(0.951)$ \\
\hline Após 2000 & $1.619 * *(0.576)$ \\
\hline Pós-sec. & $1.805^{* *}(0.691)$ \\
\hline Aluguel & $-1.043 * * *(0.355)$ \\
\hline Constante & $6.117(10.493)$ \\
\hline Observações & 31 \\
\hline $\mathbf{R}^{2}$ & 0.504 \\
\hline $\mathbf{R}^{2}$ ajustado & 0.291 \\
\hline Erro Padrão Residual & $0.549(\mathrm{df}=21)$ \\
\hline Estatística F & $2.369 * *(\mathrm{df}=9 ; 21)$ \\
\hline Nota: & $* \mathrm{p}<0.1 ; * * \mathrm{p}<0.05 ; * * * \mathrm{p}<0.01$ \\
\hline
\end{tabular}


de crimes ambientais foram "casado/união estável", "viúvo", residentes na região "após 2000" e nível de escolaridade "pós-secundário". Aquelas que contribuíram negativamente com o número de registros foram "renda per capita", faixa etária entre " 40 e 59 anos" e residentes que pagam "aluguel" (Tabela 5).

Nos crimes de dano a unidades de conservação, "viúvos" e residentes na região "após 2000" surgem como variáveis que influenciaram diretamente para aumentar o número de registros desse tipo de crime. As variáveis que contribuíram para reduzir o número de crimes ambientais foram "renda per capita", faixa etária entre "40 e 59 anos" e residentes que pagam "aluguel" (Tabela 6).

Nos registros totais de crimes de dano a unidades de conservação, o coeficiente de regressão ( $\mathrm{R}^{2}$ ajustado), ou a proporção da variância que é explicada pelo modelo, foi considerado baixo, correspondente a $29,1 \%$ e $24,3 \%$, respectivamente. Isso significa que outras variáveis, não analisadas

TABELA 6 - Regressão linear multivariada, crimes de dano a unidades de conservação/100 mil habitantes, Distrito Federal, Brasil.

\begin{tabular}{ll} 
Dano/100mil & b (erro padrão) \\
\hline Renda per capita & $-2.657^{* *}(1.029)$ \\
\hline Masculino & $12.656(8.517)$ \\
\hline $\mathbf{1 9 - 2 4 a ~ \% ~}$ & $3.047(2.206)$ \\
\hline $\mathbf{4 0 - 5 9 a} \%$ & $-5.520^{* *}(2.563)$ \\
\hline Casado/união est. & $3.691(2.766)$ \\
\hline Viúvo & $2.949^{* *}(1.358)$ \\
\hline Após 2000 & $1.774^{* *}(0.763)$ \\
\hline Pós-sec. & $2.225(0.912)$ \\
\hline Aluguel & $-1.359^{* * *}(0.468)$ \\
\hline Constante & $-41.599(35.537)$ \\
\hline
\end{tabular}

\begin{tabular}{ll}
\hline Observações & 31 \\
\hline $\mathbf{R}^{2}$ & 0.470 \\
\hline $\mathbf{R}^{2}$ ajustado & 0.243 \\
\hline Erro Padrão Residual & $0.728(\mathrm{df}=21)$ \\
\hline Estatística $\mathbf{F}$ & $2.068^{*}(\mathrm{df}=9 ; 21)$ \\
\hline Nota: & ${ }^{*} \mathrm{p}<0.1 ; * * \mathrm{p}<0.05 ; * * * \mathrm{p}<0.01$ \\
\hline
\end{tabular}


aqui, influenciam a ocorrência desse conjunto de crimes no DF.

No que se refere aos crimes contra a fauna, indivíduos com faixa etária entre " 25 e 39 anos" e com " 65 anos ou mais" foram os principais atores a influenciarem o aumento dos registros desse tipo de crime. Outras variáveis, como "renda per capita", gênero "masculino", "solteiro", "divorciados/ separados" e "residentes a menos de um ano" contribuíram para reduzir o número de registros de crimes ambientais. Em conjunto, essas variáveis foram capazes de explicar 55,5\% dos registros desse tipo de crime (Tabela 7).

O modelo de regressão multivariada calculado para os crimes contra a flora foi capaz de explicar a parcela mais significativa da variância entre os modelos obtidos. As variáveis idade entre " 19 a 24 anos", "60 a 64 anos" e residentes na região "após 2000 " influenciaram o aumento de registros de crimes, enquanto idade de "15 a 18 anos", residentes na

TABELA 7 - Regressão linear multivariada, crimes contra a fauna/100 mil habitantes, Distrito Federal, Brasil.

\begin{tabular}{ll}
$\mathbf{6 5 a}-\mathbf{m a i s} \%$ & $\mathbf{1 . 8 2 6 * * *}(\mathbf{0 . 5 9 5})$ \\
\hline Solteiro & $-4.748^{* * *}(1.492)$ \\
\hline Divorciado/separado & $-2.267 * * *(0.699)$ \\
\hline Viúvo & $-1.202(0.882)$ \\
\hline Menos 1 ano & $-0.807^{* *}(0.369)$ \\
\hline Constante & $73.555^{* * *}(24.634)$
\end{tabular}

\begin{tabular}{ll}
\hline Observaçoes & 31 \\
\hline $\mathbf{R}^{2}$ & 0.688 \\
\hline $\mathbf{R}^{2}$ ajustado & 0.555 \\
\hline Erro Padrão Residual & $0.420(\mathrm{df}=21)$ \\
\hline Estatística $\mathbf{F}$ & $5.155^{* * *}(\mathrm{df}=9 ; 21)$ \\
\hline Nota: & $* \mathrm{p}<0.1 ; * * \mathrm{p}<0.05 ; * * * \mathrm{p}<0.01$ \\
\hline
\end{tabular}


região a menos de um ano e indivíduos com nível de escolaridade "superior incompleto" influenciaram a redução desses mesmos crimes (Tabela 8).
O modelo obtido para os crimes contra o ordenamento urbano e o patrimônio cultural forneceu uma explicação moderada da proporção da

TABELA 8 - Regressão linear multivariada, crimes contra a flora/100 mil habitantes, Distrito Federal, Brasil.
Flora/100mil
b (erro padrão)

\begin{tabular}{|c|c|}
\hline $15-18 a \%$ & $-0.478 * *(0.193)$ \\
\hline $19-24 a \%$ & $1.439 *(0.740)$ \\
\hline $40-59 a \%$ & $1.776(1.083)$ \\
\hline $60-64 a \%$ & $0.997 * *(0.396)$ \\
\hline Solteiro & $1.403(0.986)$ \\
\hline Casado/união est. & $1.110(0.914)$ \\
\hline Após 2000 & $0.765 * * *(0.247)$ \\
\hline Menos 1 ano & $-0.977 * * *(0.254)$ \\
\hline Sup. incomp. & $-1.263 * *(0.469)$ \\
\hline Constante & $-16.228 * *(5.745)$ \\
\hline Observações & 31 \\
\hline $\mathbf{R}^{2}$ & 0.647 \\
\hline $\mathbf{R}^{2}$ ajustado & 0.496 \\
\hline Erro Padrão Residual & $0.287(\mathrm{df}=21)$ \\
\hline Estatística F & $4.279 * * *(\mathrm{df}=9 ; 21)$ \\
\hline Nota: & $*_{\mathrm{p}}<0.1 ; * * \mathrm{p}<0.05 ; * * * \mathrm{p}<0.01$ \\
\hline
\end{tabular}


variância. Somente duas variáveis contribuíram significativamente para esse tipo de crime: faixa etária de "40 a 59 anos" e "divorciados/separados" contribuíram para a redução dos registros desse tipo de crime (Tabela 9).
No que diz respeito à composição etária, pode-se depreender que, para o conjunto de dados avaliado, os resultados apresentaram uma relação frequentemente direta com o registro de crimes. Isso significa que não existe uma faixa etária que tenha

TABELA 9 - Regressão linear multivariada, crimes contra o ordenamento urbano e patrimônio cultural/100 mil habitantes, Distrito Federal, Brasil.

\begin{tabular}{ll}
\hline Urbano/100mil & b (erro padrão) \\
\hline Renda per cap. & $-1.190(0.890)$ \\
\hline $\mathbf{1 9 - 2 4 a ~ \% ~}$ & $2.058(1.501)$ \\
\hline $\mathbf{2 5 - 3 9 a} \%$ & $-1.214(0.845)$ \\
\hline $\mathbf{4 0 - 5 9 a} \%$ & $-5.902^{* * *}(1.934)$ \\
\hline Casado/união est. & $2.555(1.808)$ \\
\hline Divorciado/separado & $-1.663^{* *}(0.711)$ \\
\hline Após 2000 & $0.483(0.390)$ \\
\hline Menos 1 ano & $-0.692(0.510)$ \\
\hline Sup. incomp. & $1.940(1.126)$ \\
\hline Pós-sec. & $1.401(1.013)$ \\
\hline Constante & $14.015(9.585)$ \\
\hline
\end{tabular}

\begin{tabular}{ll}
\hline Observações & 31 \\
\hline $\mathbf{R}^{2}$ & 0.615 \\
\hline $\mathbf{R}^{2}$ ajustado & 0.423 \\
\hline Erro Padrão Residual & $0.492(\mathrm{df}=20)$ \\
\hline Estatística $\mathbf{F}$ & $3.201 * *(\mathrm{df}=10 ; 20)$ \\
\hline Nota: & ${ }^{*} \mathrm{p}<0.1 ; * * \mathrm{p}<0.05 ; * * * \mathrm{p}<0.01$ \\
\hline
\end{tabular}


influenciado de forma diferenciada a ocorrência de crimes ambientais. A exceção é a faixa etária de "40 a 59 anos", identificada como a variável independente que mais frequentemente influenciou a redução de registros de crimes ambientais. A faixa etária entre "15 e 18 anos" também influenciou a redução de registros de crimes, resultado esperado pelo fato de menores de 18 anos serem criminalmente inimputáveis. Dessa forma, a teoria da atividade de rotina, no que diz respeito às variáveis preditoras "faixa etária" avaliadas por Hirschi \& Gottfredson em 1983, poderia ser aplicada aos crimes ambientais, mas com resultado distinto do obtido no trabalho desses autores, em que a curva idade-crime identificou a presença de potenciais infratores na parcela da população com idades entre 15 e 24 anos.

O estado civil dos habitantes se mostrou uma variável preditora pouco consistente, por vezes contribuindo diretamente, outras inversamente para os registros de crimes ambientais no DF. A teoria da atividade de rotina não poderia então ser aplicada para essas variáveis.

A imigração recente, aqui considerada como aqueles que imigraram para o Distrito Federal (DF) após do ano 2000, explicou o aumento do número registrado de crimes ambientais em três dos cinco modelos obtidos. Conforme dados do IBGE (2010), o DF atingiu o primeiro milhão de habitantes entre 1960 e 1990, e o segundo milhão de habitantes na década seguinte, entre 1990 e 2000. Segundo estimativa da Pesquisa Distrital por Amostra de Domicílios (PDAD), em 2015 o DF estava próximo em atingir seu terceiro milhão de habitantes (CODEPLAN, 2016). Os dados revelam um processo de imigração descontrolada entre 1990 e 2000 (um milhão de habitantes em 10 anos), que atenuou brevemente entre 2000 e 2015 (quase um milhão de habitantes em 15 anos). O desenvolvimento de identidade e de organização comunitária, que resulta em menor número de crimes, demanda tempo para ser construído (Sampson \& Groove, 1989).

Por outro lado, os residentes a menos de um ano no DF influenciaram a redução dos registros de crimes contra a fauna e contra a flora. Segundo as aplicações contemporâneas da teoria da desorganização social, a rotatividade populacional é medida utilizando o percentual de residentes que se mudaram para a região no último ano e o percentual de unidade alugadas (Andrensen, 2011). Como os locatários tendem a ser mais transientes e menos familiarizados com a região que os proprietários, pode-se inferir que são menos propensos a cometerem delitos ambientais onde residem.

Teoricamente, o maior nível de educação formal influenciaria a consciência de uma comunidade sobre a importância de um ambiente preservado, uma vez que a educação conservacionista criaria bons comportamentos perante a natureza (Tanner, 1978). Todavia, o nível de escolaridade "pós-secundário" apresentou um resultado diverso do esperado, com uma relação direta com o aumento dos registros de crimes ambientais totais. No entanto, o $\mathrm{R}^{2}$ ajustado do modelo obtido para "crimes ambientais totais" mostrou-se baixo, indicando que outras variáveis não avaliadas estão influenciando esta análise, tornando o resultado encontrado pouco expressivo.

A Lei de Crimes Ambientais (Lei n. ${ }^{\circ}$ 9.605/1998), que inovou ao erigir a pessoa jurídica à condição de sujeito ativo da relação processual penal, observa que a responsabilidade das pessoas jurídicas não exclui a responsabilidade das pessoas físicas autoras, coautoras ou partícipes do mesmo fato (Brasil, 1998). Para tanto, a pessoa jurídica 
pode ser desconsiderada sempre que se tornar obstáculo ao ressarcimento de prejuízos provocados. A imputação à pessoa física, na figura do diretor, administrador, membro de conselho e de órgão técnico, auditor, gerente, preposto ou mandatário da pessoa jurídica pode ser a chave para compreender essa relação direta observada entre o nível de escolaridade "pós-secundário" e o número de registros totais de crimes ambientais no DF.

A variável "renda per capita" surgiu como preditora em três das análises realizadas. Seguindo o que se defende em estudos sobre criminalidade violenta, nos crimes ambientais a relação entre renda e registros de crimes é inversa. Esse resultado confronta as conclusões defendidas por Cohen $\&$ Felson (1979) sobre as atividades de rotina, em que, além de sinônimos de colapso social, os crimes podem ser tomados como subproduto de liberdade e prosperidade. No entanto, o resultado aqui obtido encontra alicerce na teoria defendida por Blau \& Blau (1982), em que a criminalidade seria uma consequência da privação socioeconômica relativa do indivíduo. Os bens materiais obtidos como fruto dos crimes cometidos contra a natureza em muitos casos tornam-se fonte de renda, única ou extra, para os autores dos delitos. Muitos dos tipos penais descritos na Lei n. ${ }^{\circ}$ 9.605/98 referem-se a atos de comercialização de bens naturais.

A maioria dos tipos penais contidos na Lei de Crimes Ambientais (Lei n. ${ }^{\circ}$ 9.605/1998) é considerada de menor potencial ofensivo, ou seja, possuem pena máxima cominada não superior a dois anos ou multa. No entanto, existem duas possibilidades de conversão da pena privativa de liberdade em penas mais brandas ou até a declaração de extinção do direito do Estado em punir o autor do crime, caso seja comprovada a impossibilidade de reparação do dano, ou se, após sucessivos laudos de constatação de reparação do dano, for concluído que o acusado tomou todas as providências para a necessária reparação do dano.

Com penas restritivas de liberdade baixas e com uma gama de situações que levam à possibilidade de perdão pelo crime, é previsível concluir que o crime ambiental compensa sob esse cenário. Daqui depreende-se que diferentes resultados conduzem a uma mesma conclusão defendida por Beato Filho (1998): a materialização do crime seria consequência de uma ponderação racional dos custos e benefícios acerca da viabilidade do delito.

\section{Considerações finais}

As variáveis que mais significativamente contribuíram para reduzir o número de registros de crimes ambientais no Distrito Federal entre os anos de 2013 e 2016 foram faixa etária entre " 40 e 59 anos" e a renda per capita. De forma menos incisiva, observou-se a existência de uma relação entre e os locatários e residentes de imóveis a menos de um ano e um menor número de registros de crimes ambientais. A imigração posterior ao ano 2000 contribuiu significativamente para aumentar o número de registros de crimes ambientais no DF entre 2013 e 2016, condizente com o escopo defendido pela teoria da desorganização social.

Os resultados encontrados neste trabalho sugerem que contextos de privação social e econômica, apesar de importantes, não são os únicos determinantes para a ocorrência de crimes ambientais, conforme os dados e as teorias avaliadas. A frequente relação com as variáveis relacionadas à imigração sugere uma possível aplicação da teoria 
da desorganização social aos crimes ambientais, já que moradores recentes refletem a incapacidade da comunidade se organizar em prol do controle comunitário.

\section{Agradecimentos}

Agradecemos à Direção Geral da Polícia Civil do Distrito Federal, na pessoa do Dr. Eric Seba, pelo apoio, tornando possível a execução deste trabalho.

\section{Referências}

Andrensen, M. A. Estimating the probability of local crime clusters: The impact of immediate spatial neighbors. Journal of Criminal Justice, 39, 394-404, 2011. doi: 10.1016/j. jcrimjus.2011.05.005

Batella, W. B.; Diniz, A. M. A. Análise espacial dos condicionantes da criminalidade violenta no Estado de Minas Gerais. Sociedade \& Natureza, 22(1), 151-163, 2010.

Beato, C. C.; Reis, I. A. Desigualdade, desenvolvimento socioeconômico e crime. In: Henriques, R. (Org.). Desigualdade e Pobreza no Brasil. Rio de Janeiro: IPEA, p. 385 - 404, 2000.

Beato Filho, C. C. Determinantes da Criminalidade em Minas Gerais. Revista Brasileira de Ciências Sociais, 13(37), 74-89, 1998. doi: 10.1590/S0102-69091998000200004

Blau, J.; Blau, P. M. The cost of inequality: metropolitan structure and violent crime. American Sociological Review, 47(1), 114-129, 1982. doi: 10.2307/2095046

Brasil. Lei $n^{\circ}$ 9.605, de 12 de fevereiro de 1998. Dispõe sobre as sanções penais e administrativas derivadas de condutas e atividades lesivas ao meio ambiente, e dá outras providências. Brasília: DOU de 13/2/1998.

CODEPLAN - Companhia de Planejamento do Distrito Federal. Pesquisa Distrital por Amostra de Domicílios - PDAD - 2013/14, 2014. Disponível em: <http://www. codeplan.df.gov.br/component/content/article/261-pesquisas-socioeconomicas/294-PDAD-2013.html>. Acesso em: jan. 2017.

CODEPLAN - Companhia de Planejamento do Distrito Federal. Pesquisa Distrital por Amostra de Domicílios - PDAD - 2015/16, 2016. Disponível em: <http://www. codeplan.df.gov.br/component/content/article/261-pesquisas-socioeconomicas/319-pdad-2015.html>. Acesso em: jan. 2017.

Cohen, L. E.; Felson, M. Social Change and Crime Rate Trends: A Routine Activity Approach. American Sociological Review, 44(4), 588-608, 1979.

Durkheim, E. Da divisão do trabalho social. São Paulo: WMF Martins Fontes, $4^{\mathrm{a}}$ ed., 2010.

Gotelli, N. J.; Ellison, A. M. Principios de estatística em ecologia. Artmed: Porto Alegre, $1^{\mathrm{a}}$ ed., 2011.

Hirschi, T.; Gottfredson, M. Age and the explanation of crime. American Journal of Sociology, 89, 552-584, 1983.

IBGE - Instituto Brasileiro de Geografia e Estatística. Censo Demográfico 2010: Séries históricas, 2010. Disponível em: $<$ https://www.ibge.gov.br/estatisticas-novoportal/sociais/ populacao/9662-censo-demografico-2010.html?\&t=series-historicas>. Acesso em: dez. 2017.

Kasarda, J. D.; Janowitz, M. Community Attachment in Mass Society. American Sociological Review, 39(3), 328339, 1974.

Legendre P.; Legendre L. Numerical ecology: Developments in Environmental Modelling. United Kingdom: Elsevier, 2012.

Milaré, E. Direito do Ambiente. São Paulo: Revista dos Tribunais, 9. ed. rev. atual. e ampl., 2014.

Molina, A. G. P.; Gomes, L. F. Criminologia. São Paulo: Revista dos Tribunais, 5. ed. rev. e atual., 2007.

Parker, R. N.; Smith, M. D. Deterrence, Poverty, and Type of Homicide. American Journal of Sociology, 85(3), 614$624,1979$.

Penteado Filho, N. S. Manual esquemático de criminologia. São Paulo: Saraiva, 2. ed., 2012. 
Sampson, R. J.; Groves, W. B. Community structure and crime: Testing social-disorganization theory. American Journal of Sociology, 4, 774-802, 1989.

Shaw, C. R.; McKay, H. D. Juvenile Delinquency and Urban Areas. In: Andrensen, T. L. (Ed.). Understanding Deviance: Connecting Classical and Contemporary Perspectives. New York, NY: Routledge, 106-127, 2014.

Tanner, R.T. Educação ambiental. São Paulo: Summus/ Edusp, 1978.

Taylor, I.; Walton, P.; Young, J. Criminologia crítica, São Paulo: Graal, $1^{\text {a }}$ ed., 1980.

White, R. Crimes Against Nature: environmental criminology and ecological justice. Devon: Willan Publishing, 2008. 